\title{
Vorschriften in der Veranstaltungstechnik
}

Die wichtigsten Grundlagen für den Umgang, die Verwendung und den Einsatz von Veranstaltungstechnik sind in Unfallverhütungsvorschriften der Unfallversicherungsträger zu finden.

Der Bau und Betrieb von Versammlungsstätten ist in den Landesbauordnungen und insbesondere der Versammlungsstättenverordnung bzw. den Sonderbauverordnungen für Versammlungsstätten der jeweiligen Bundesländer geregelt.

Mit der stetigen Weiterentwicklung in der Technik werden die jeweiligen Vorschriften und Gesetze fortgeschrieben und angepasst.

Grundsätzlich wird unterschieden zwischen

- den Gesetzen und Verordnungen, die bindenden Charakter besitzen und

- den technischen Regeln, die empfehlen in welcher Art die jeweiligen Ausführungen umgesetzt werden sollen.

Neben den allgemein für jede Branche geltenden gesetzlichen Regelungen für Mitarbeiter in gewerblicher Produktion und Dienstleistung, finden spezifische Festlegungen Anwendung, die alle Beteiligten und Sachwerte bei Veranstaltungen in besonderem Maße schützen sollen.

Die Broschüre der Deutschen Eventakademie zur Planung von Veranstaltungen gibt hier einen umfangreichen Überblick über die Rechtsquellen (Lohmann o. J.). 